\title{
The structure-based design of zinc finger nucleases can facilitate genomic editing
}

A recent study of significant research value carried out at the State Key Laboratory of Biocontrol, School of Life Sciences, Sun Yat-Sen University, Guangzhou, China, and published in Issue 54 of Science China Life Sciences described a novel method using FoldX force field based protein modeling that can be applied in zinc finger nucleases design.

Zinc finger nucleases (ZFNs) can actively introduce a double-strand break (DSB) at target loci in eukaryotic genomes and this can improve the efficiency of gene knock-out or knock-in by several orders of magnitude. Therefore, ZFN is becoming a promising novel tool for genomic editing. However, most current strategies for developing zinc finger nucleases with customized sequence specificities require the construction of numerous tandem arrays of zinc finger proteins (ZFPs), and the subsequent large-scale in vitro validation of their DNA binding affinities and specificities using bacterial selection. The labor and expertise required in this complex process limits the broad adoption of the ZFN technology.

In this work, an effective computational assisted design strategy was introduced to lower the complexity of the production of a pair of functional ZFNs. Dr. He and Prof. Chen used the FoldX force field to build 3D models of 420 ZFP-DNA complexes based on zinc finger arrays developed by the Zinc Finger Consortium using OPEN (oligomerized pool engineering). Using nonlinear and linear regression analysis, they found that the calculated protein-DNA binding energy in a modeled ZFP-DNA complex strongly correlates to the failure rate of the zinc finger array to show significant ZFN activity in human cells. In their models, less than 5\% of the three-finger arrays with calculated protein-DNA binding energies lower than $-13.132 \mathrm{kcal} \mathrm{mol}^{-1}$ failed to form active ZFNs in human cells. By contrast, for arrays with calculated protein-DNA binding energies higher than $-5 \mathrm{kcal} \mathrm{mol}^{-1}$, as many as $40 \%$ of them lacked ZFN activity in human cells. Therefore, the FoldX force field based ZFN design strategy can be effectively used to reduce the failure rate and to increase efficiency in producing customized ZFNs.

"This is a novel strategy for designing customized ZFN pairs. It enables us to predict whether a candidate ZFN can function well in mammalian cells by calculating the protein-DNA binding energy within the modeled complex." was the opinion of one journal reviewer. "The authors tried a new method based on the FlodX force field to overcome the complex screening process in ZFN design. It appeared to be helpful to the rapidly developed ZFN technology." commented another reviewer.

The authors are affiliated to The State Key Laboratory of Biocontrol, School of Life Sciences, Sun Yat-Sen University, Guangzhou, China. The group mainly focuses on unraveling the genetic basis of economic traits and disease resistant capabilities in swine using comprehensive strategies that include the generation of transgenic or gene knock-out pig models.

Funding for the project was provided by the National Natural Science Foundation of China (30901018) and the China Postdoctoral Science Foundation (201003388).

See the article: He Z Y, Mei G, Zhao C P, et al. Potential application of FoldX force field based protein modeling in zinc finger nucleases design. Sci China Life Sci, 2011, 54: 442-449, doi: 10.1007/s11427-011-4159-9

Open Access This article is distributed under the terms of the Creative Commons Attribution License which permits any use, distribution, and reproduction in any medium, provided the original author(s) and source are credited. 